\title{
Towards the bounce inflationary gravitational wave
}

\author{
Hai-Guang $\mathbf{L i}^{1, \mathrm{a}}$, Yong Cai ${ }^{1, \mathrm{~b}}$, Yun-Song Piao ${ }^{1,2, \mathrm{c}}$ \\ ${ }^{1}$ School of Physical Sciences, University of Chinese Academy of Sciences, Beijing 100049, China \\ ${ }^{2}$ Institute of Theoretical Physics, Chinese Academy of Sciences, P.O. Box 2735, Beijing 100190, China
}

Received: 23 October 2016 / Accepted: 2 December 2016 / Published online: 20 December 2016

(C) The Author(s) 2016. This article is published with open access at Springerlink.com

\begin{abstract}
In the bounce inflation scenario, the inflation is singularity-free, while the advantages of inflation are preserved. We analytically calculate the power spectrum of its primordial gravitational waves (GWs), and show a universal result including the physics of the bounce phase. The spectrum acquires a cutoff at large scale, while the oscillation around the cutoff scale is quite drastic, which is determined by the details of bounce. Our work highlights that the primordial GWs at large scale may encode the physics of the bounce ever happened at about $\sim 60$ efolds before inflation.
\end{abstract}

\section{Introduction}

Inflation [1-4] is the paradigm of the early universe. However, it is confronted with the so-called "initial singularity problem", since the inflation itself is past-incomplete [5]. The solution to this problem must involve something occurring before inflation. One possibility is the so-called bounce inflation scenario [6-8], see also [9-16], in which initially the universe is contracting, and after the bounce, the inflation starts.

Recently, the Planck collaboration $[17,18]$ have observed the power deficit of the CMB TT-mode spectrum at large scale. This inspired theorists to think over the physics of the pre-inflation, about $\sim 60$ efolds before the inflation during which the evolutions of largest scale perturbations are involved, e.g., [19]. It is interesting that the pre-inflationary physics suggested by the large-scale power deficit might be relevant with the initial singularity problem. The bounce universe, as the solution to this problem, has a long history of study; see [20,21] for reviews. In Refs. [6-10], it has been discovered that in the bounce inflation scenario the largescale anomalies of CMB TT spectrum may be explained nat-

\footnotetext{
a e-mail: lihaiguang14@mails.ucas.ac.cn

b e-mail: caiyong13@mails.ucas.ac.cn

c e-mail: yspiao@ucas.ac.cn
}

urally. This achievement makes the bounce inflation acquire increasing attention.

Primordial GWs are also a product of inflation. Recently, lots of experiments aiming at detecting GWs have been implemented or will be implemented, which will bring us a new epoch to understand the physics of inflation scenario; see e.g., [22]. There are also other designs to explain the large-scale power deficit of the scalar perturbation spectrum, the suppression is attributed to the rapid rolling of the scalar field. However, the primordial GWs are independent of the dynamics of the scalar field, which thus may be used to identify the physics of the pre-inflationary background.

Recently, the nonsingular bounce without modifying gravity has been implemented, which may be ghost-free, e.g., [23-28], with possible embedding into supergravity $[29,30]$. Thus the features of the primordial GWs at large scale might tell us if such a bounce has ever happened at about $\sim 60$ efolds before inflation. Moreover, it might also help us to speculate the property of the cyclic universe [3139] with such a nonsingular bounce.

In this paper, we analytically calculate the bounce inflationary GWs. The resulting spectrum is written with the recursive Bogoliubov coefficients including the physics of the bounce phase. We also show that our analytic result is completely consistent with the numerical plotting for a realistic model of bounce inflation.

\section{Overview of bounce inflation scenario}

In the bounce inflation scenario, inflation is singularity-free, while the advantages of inflation are preserved, which simply and naturally explains the universe we live in.

The idea of bounce inflation showed itself first in Refs. [68], which explained the large-scale suppression of a scalar perturbation spectrum observed by WMAP. Based on the quintom bounce [40], Ref. [41] investigated the evolution of the primordial perturbations in detail, and recently, Qiu and 
Wang [15] and Wan et al. [16] have constructed a realistic model of bounce inflation without instabilities by applying the higher-derivative operator.

The bounce inflation also has been implemented in the positively curved background [11,12], or by modifying gravity $[9,13]$. The matching of the perturbation through the bounce with the modified gravity was discussed in [38].

Recently, Liu et al. [10] have found that the bounce inflation brings about not only the large-scale power deficit of $\mathrm{CMB}$, but also a large hemispherical power asymmetry, as implied by Planck. Current bounds on the primordial GWs have been used to constrain the bounce inflation [14]. However, Refs. [14] used a step-like parameterization of the primordial GW spectrum, based on [6-8], which is only a rough estimate missing the effect of the bounce. Recently, the detecting of primordial GWs has been under way, which will possibly tell us more on inflation and its origin. Therefore, it is significant to have a full study for the bounce inflationary GWs.

\section{Bounce inflationary GWs: analytical result}

Tensor perturbation $\gamma_{i j}$ satisfies $\gamma_{i i}=0$ and $\partial_{i} \gamma_{i j}=0$. Its action is

$S_{\gamma}^{(2)}=\int \mathrm{d} \eta \mathrm{d}^{3} x \frac{a^{2}}{8}\left[\left(\frac{\mathrm{d} \gamma_{i j}}{\mathrm{~d} \eta}\right)^{2}-\left(\vec{\nabla} \gamma_{i j}\right)^{2}\right]$,

where $\eta=\int \mathrm{d} t / a$ and $M_{P}^{2}=1$.

The Fourier series of $\gamma_{i j}$ is

$h_{i j}(\eta, \mathbf{x})=\int \frac{\mathrm{d}^{3} k}{(2 \pi)^{3}} e^{-i \mathbf{k} \cdot \mathbf{x}} \sum_{\lambda=+, \times} \hat{h}_{\lambda}(\eta, \mathbf{k}) \epsilon_{i j}^{(\lambda)}(\mathbf{k})$,

in which $\hat{h}_{\lambda}(\eta, \mathbf{k})=h_{\lambda}(\eta, k) a_{\lambda}(\mathbf{k})+h_{\lambda}^{*}(\eta,-k) a_{\lambda}^{\dagger}(-\mathbf{k})$, polarization tensors $\epsilon_{i j}^{(\lambda)}(\mathbf{k})$ satisfy $k_{j} \epsilon_{i j}^{(\lambda)}(\mathbf{k})=0, \epsilon_{i i}^{(\lambda)}(\mathbf{k})=$ 0 , and $\epsilon_{i j}^{(\lambda)}(\mathbf{k}) \epsilon_{i j}^{*\left(\lambda^{\prime}\right)}(\mathbf{k})=\delta_{\lambda \lambda^{\prime}}, \epsilon_{i j}^{*(\lambda)}(\mathbf{k})=\epsilon_{i j}^{(\lambda)}(-\mathbf{k})$, the annihilation and creation operators $a_{\lambda}(\mathbf{k})$ and $a_{\lambda}^{\dagger}\left(\mathbf{k}^{\prime}\right)$ satisfy $\left[a_{\lambda}(\mathbf{k}), a_{\lambda^{\prime}}^{\dagger}\left(\mathbf{k}^{\prime}\right)\right]=\delta_{\lambda \lambda^{\prime}} \delta^{(3)}\left(\mathbf{k}-\mathbf{k}^{\prime}\right)$. The equation of motion for $u(\eta, k)$ is (Fig. 1)

$\frac{\mathrm{d}^{2} u}{\mathrm{~d} \eta^{2}}+\left(k^{2}-\frac{a^{\prime \prime}}{a}\right) u=0$,

where $u(\eta, k)=\frac{a h_{\lambda}(\eta, k)}{2}$. The spectrum of primordial GWs is

$P_{T}=\frac{k^{3}}{2 \pi^{2}} \sum_{\lambda=+, \times}\left|h_{\lambda}\right|^{2}=\frac{4 k^{3}}{\pi^{2}} \cdot \frac{1}{a^{2}}|u|^{2}, \quad a H / k \gg 1$.

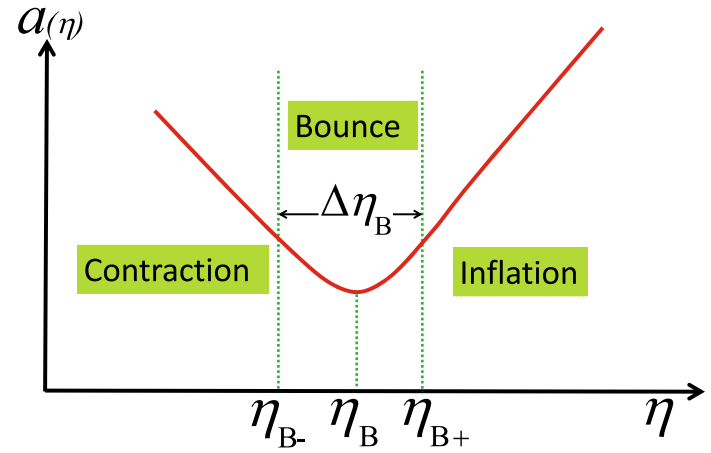

Fig. 1 The illustration of the bounce inflation scenario. The $\eta_{B}$ - is the beginning time of bounce phase, at which $\mathcal{H}_{B-}=a H_{B-}<0$ and $\dot{H}_{B-}=0 . \eta_{B}$ is the so-called bounce point, at which $H=0 . \eta_{B+}$ is the end time of bounce phase, at which $\mathcal{H}_{B+}=a H_{B+}>0$ and $\dot{H}_{B+}=0$. After $\eta_{B+}$, inflation started

\subsection{The contracting phase}

The contracting phase is the evolution with $H<0$ and $\dot{H}<$ 0 . It ends at $\eta_{B}$ - when $\dot{H}=0$. Hereafter, $\dot{H}>0$, the bounce starts.

The background can be parameterized as

$a_{c}(\eta)=a_{B-}\left(\frac{\eta-\tilde{\eta}_{B-}}{\eta_{B-}-\tilde{\eta}_{B-}}\right)^{\frac{1}{\epsilon_{C}-1}}$,

where $\tilde{\eta}_{B-}=\eta_{B-}-\left[\left(\epsilon_{C}-1\right) \mathcal{H}_{B-}\right]^{-1}$, noting the continuities of $a$ and $\mathcal{H}$ at $\eta_{B-}$, and $\epsilon_{C}=-\dot{H} / H^{2}$ and $\mathcal{H}_{B-}$ is the comoving Hubble parameter at $\eta_{B-}$. The initial state is the Minkowski vacuum

$u_{k}=\frac{1}{\sqrt{2 k}} e^{i k \eta}$.

Thus the solution of Eq. (3) is

$u_{k}=\frac{\sqrt{\pi\left|\eta-\tilde{\eta}_{B-}\right|}}{2} c_{1,1} H_{\nu_{1}}^{(1)}\left(k\left|\eta-\tilde{\eta}_{B-}\right|\right)$,

where $v_{1}=\frac{\epsilon_{c}-3}{2\left(\epsilon_{c}-1\right)}$.

\subsection{The bounce phase}

The bounce phase is the evolution with $\dot{H}>0$. The Hubble parameter is parameterized as $[15,41]$

$H=\alpha\left(t-t_{B}\right)$,

with $\alpha M_{P}^{2} \ll 1$. Thus we have

$a \simeq a_{B} e^{\frac{1}{2} \alpha\left(t-t_{B}\right)^{2}} \simeq a_{B}\left[1+\frac{\alpha}{2}\left(t-t_{B}\right)^{2}\right]$,

where $a=a_{B}$ at $t=t_{B}$. Equation (8) indicates that the bounce phase is actually a superinflation phase with $H$ 
rapidly increasing. In Ref. [9], it was argued that this phase results in the large-scale power deficit in CMB. However, we will show that in a bounce inflation scenario this power deficit is actually attributed to the contraction before the bounce.

The continuities of $a$ and $\mathcal{H}$ at $\eta_{B-}$ and $\eta_{B+}$ suggest

$\mathcal{H}_{B+}=\mathcal{H}_{B-}+\alpha a_{B}^{2} \triangle \eta_{B}$.

We have $\mathcal{H}_{B+}=\frac{\alpha a_{B}^{2} \Delta \eta_{B}}{2}$ for $\mathcal{H}_{B+} \simeq-\mathcal{H}_{B-} . \mathcal{H}_{B+}$ is actually the comoving Hubble parameter at the beginning time of the inflation. $\alpha$ and $\Delta \eta_{B}=\eta_{B+}-\eta_{B-}$ encode the physics of the bounce phase. Actually, since $a \simeq a_{B}$ during the bounce, we can find $\eta-\eta_{B}=a_{B}^{-1}\left(t-t_{B}\right)$. Thus Eq. (3) becomes

$u_{k}^{\prime \prime}+\left(k^{2}-\alpha a_{B}^{2}\right) u_{k}=0$.

Its solution is

$u_{k}=c_{2,1} e^{l\left(\eta-\eta_{B}\right)}+c_{2,2} e^{-l\left(\eta-\eta_{B}\right)}$,

where $l \equiv \sqrt{\alpha a_{B}^{2}-k^{2}}$.

\subsection{The inflationary phase}

The universe will inflate after $\eta_{B+}$. The background is parameterized as

$a_{\text {inf }}(\eta)=a_{B+}\left(\frac{\eta-\tilde{\eta}_{B+}}{\eta_{B+}-\tilde{\eta}_{B+}}\right)^{\frac{1}{\epsilon_{\text {inf }}-1}}$.

Here, $\tilde{\eta}_{B+}=\eta_{B+}-\left[\left(\epsilon_{\text {inf }}-1\right) \mathcal{H}_{B+}\right]^{-1}$, noting the continuities of $a$ and $\mathcal{H}$ at $\eta_{B+}$, and $\epsilon_{\text {inf }}=-\dot{H} / H^{2} . H_{B+}=\mathcal{H}_{B+} / a$ sets the scale of inflation after the bounce, $H=H_{B+}$.

Thus the solution of Eq. (3) is

$$
\begin{aligned}
u_{k}= & \frac{\sqrt{\pi\left|\eta-\tilde{\eta}_{B+1}\right|}}{2}\left[c_{3,1} H_{v_{2}}^{(1)}\left(k\left|\eta-\tilde{\eta}_{B+}\right|\right)+\right. \\
& \left.\times c_{3,2} H_{v_{2}}^{(2)}\left(k\left|\eta-\tilde{\eta}_{B+}\right|\right)\right]
\end{aligned}
$$

where $v_{2}=\frac{\epsilon_{\text {inf }}-3}{2\left(\epsilon_{\text {inf }}-1\right)}$.

\subsection{The spectrum of primordial GWs}

According to (4), if $\epsilon_{\text {inf }}=0$ we find

$P_{T}=\frac{2 H_{B+}^{2}}{\pi^{2}}\left|c_{31}-c_{32}\right|^{2}=P_{T}^{i n f}\left|c_{31}-c_{32}\right|^{2}$,

where $P_{T}^{i n f}=\frac{2 H_{B+}^{2}}{\pi^{2}}$ is the standard result of the slow-roll inflation.

The perturbation $\gamma_{i j}$ and its time derivative should be continuous through the match surface. This suggests that we could write the coefficients recursively as

$$
\left(\begin{array}{l}
c_{3,1} \\
c_{3,2}
\end{array}\right)=\mathcal{M}^{(3,2)} \times \mathcal{M}^{(2,1)} \times\left(\begin{array}{l}
c_{1,1} \\
c_{1,2}
\end{array}\right),
$$

where the matric $\mathcal{M}^{(2,1)}$ is

$$
\begin{aligned}
\mathcal{M}_{11}^{(2,1)}= & e^{-l y_{1}} \frac{\sqrt{\pi x_{1}}}{8 l}\left\{k\left[-H_{v_{1}-1}^{(1)}\left(k x_{1}\right)+H_{v_{1}+1}^{(1)}\left(k x_{1}\right)\right]\right. \\
& \left.+2\left[-\frac{\nu_{1}}{x_{1}}+l+\alpha a_{B}^{2} y_{1}\right] H_{v_{1}}^{(1)}\left(k x_{1}\right)\right\}, \\
\mathcal{M}_{12}^{(2,1)}= & e^{-l y_{1}} \frac{\sqrt{\pi x_{1}}}{8 l}\left\{k\left[-H_{v_{1}-1}^{(2)}\left(k x_{1}\right)+H_{v_{1}+1}^{(2)}\left(k x_{1}\right)\right]\right. \\
& \left.+2\left[-\frac{\nu_{1}}{x_{1}}+l+\alpha a_{B}^{2} y_{1}\right] H_{v_{1}}^{(2)}\left(k x_{1}\right)\right\}, \\
\mathcal{M}_{21}^{(2,1)}= & e^{l y_{1}} \frac{\sqrt{\pi x_{1}}}{8 l}\left\{k\left[H_{v_{1}-1}^{(1)}\left(k x_{1}\right)-H_{v_{1}+1}^{(1)}\left(k x_{1}\right)\right]\right. \\
& \left.+2\left[-\frac{\nu_{1}}{x_{1}}+l-\alpha a_{B}^{2} y_{1}\right] H_{v_{1}}^{(1)}\left(k x_{1}\right)\right\}, \\
\mathcal{M}_{22}^{(2,1)}= & e^{l y_{1}} \frac{\sqrt{\pi x_{1}}}{8 l}\left\{k\left[H_{v_{1}-1}^{(2)}\left(k x_{1}\right)-H_{v_{1}+1}^{(2)}\left(k x_{1}\right)\right]\right. \\
& \left.+2\left[-\frac{\nu_{1}}{x_{1}}+l-\alpha a_{B}^{2} y_{1}\right] H_{v_{1}}^{(2)}\left(k x_{1}\right)\right\},
\end{aligned}
$$

and $\mathcal{M}^{(3,2)}$ is

$$
\begin{aligned}
\mathcal{M}_{11}^{(3,2)}= & \frac{i \sqrt{\pi x_{2}}}{4} e^{l y_{2}}\left\{k\left[H_{v_{2}-1}^{(2)}\left(k x_{2}\right)-H_{v_{2}+1}^{(2)}\left(k x_{2}\right)\right]\right. \\
& \left.+2\left[\frac{\nu_{2}}{x_{2}}+l-\alpha a_{B}^{2} y_{2}\right] H_{v_{2}}^{(2)}\left(k x_{2}\right)\right\}, \\
\mathcal{M}_{12}^{(3,2)}= & \frac{i \sqrt{\pi x_{2}}}{4} e^{-l y_{2}}\left\{k\left[H_{v_{2}-1}^{(2)}\left(k x_{2}\right)-H_{v_{2}+1}^{(2)}\left(k x_{2}\right)\right]\right. \\
& \left.+2\left[\frac{\nu_{2}}{x_{2}}-l-\alpha a_{B}^{2} y_{2}\right] H_{v_{2}}^{(2)}\left(k x_{2}\right)\right\}, \\
-\mathcal{M}_{21}^{(3,2)}= & \frac{i \sqrt{\pi x_{2}}}{4} e^{l y_{2}}\left\{k\left[H_{v_{2}-1}^{(1)}\left(k x_{2}\right)-H_{v_{2}+1}^{(1)}\left(k x_{2}\right)\right]\right. \\
& \left.+2\left[\frac{\nu_{2}}{x_{2}}+l-\alpha a_{B}^{2} y_{2}\right] H_{v_{2}}^{(1)}\left(k x_{2}\right)\right\}, \\
-\mathcal{M}_{22}^{(3,2)}= & \frac{i \sqrt{\pi x_{2}}}{4} e^{-l y_{2}}\left\{k\left[H_{v_{2}-1}^{(1)}\left(k x_{2}\right)-H_{v_{2}+1}^{(1)}\left(k x_{2}\right)\right]\right. \\
& \left.+2\left[\frac{\nu_{2}}{x_{2}}-l-\alpha a_{B}^{2} y_{2}\right] H_{v_{2}}^{(1)}\left(k x_{2}\right)\right\},
\end{aligned}
$$

with the definitions $x_{1}=\left|\eta_{B-}-\tilde{\eta}_{B-}\right|, y_{1}=\left(\eta_{B-}-\eta_{B}\right)$, $x_{2}=\left|\eta_{B+}-\tilde{\eta}_{B+}\right|$ and $y_{2}=\left(\eta_{B+}-\eta_{B}\right)$.

The effects of pre-inflationary phases are encoded in $\mathcal{M}^{(3,2)}$ and $\mathcal{M}^{(2,1)}$. Here, we set the slow-roll parameter of inflation $\epsilon_{\text {inf }} \simeq 0$, thus $P_{T}$ is only relevant with the parameters, $\alpha, \Delta \eta_{B}$, and $\epsilon_{c}$, noting that $\epsilon_{c} \geq 3$ must be satisfied to avoid the cosmic anisotropy problem [42]. We plot $P_{T}$ in Figs. 2 and 3 by altering the values of different parameters. We see that, for $k>\mathcal{H}_{B+}, P_{T} \sim k^{0}$ with a damped 


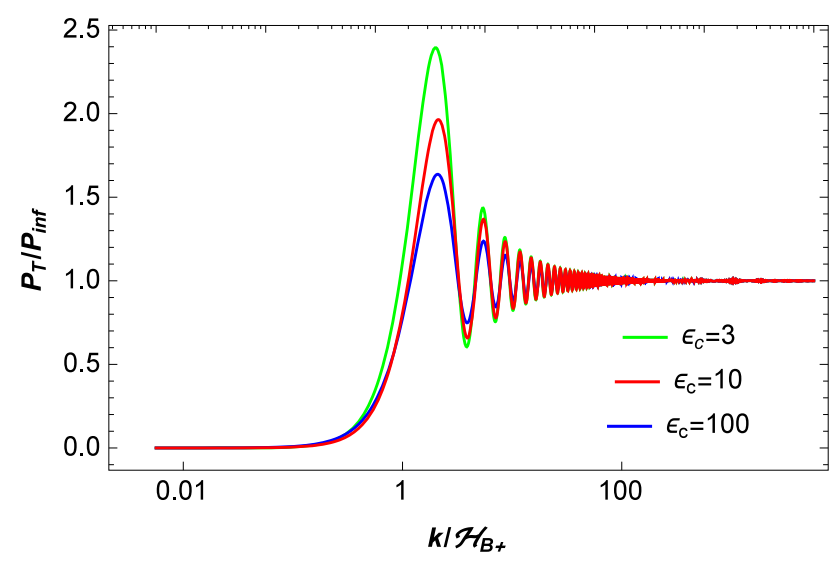

Fig. $2 P_{T}$ in Eq. (15) for different $\epsilon_{c}$. We set $\Delta \eta=0.2 / \mathcal{H}_{B+}$ and $\alpha=1.8 \times 10^{-3} \mathcal{H}_{B+}^{2}$

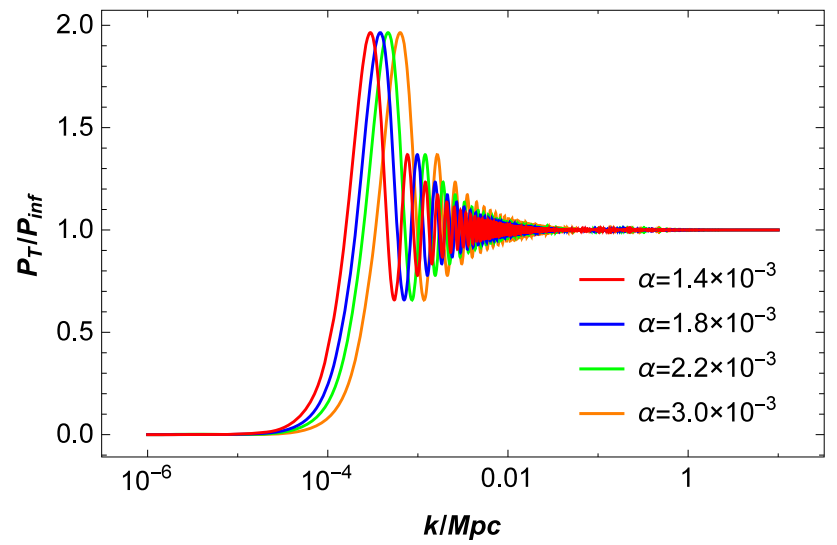

Fig. $3 P_{T}$ in Eq. (15) for different $\alpha$ in units of $\mathcal{H}_{B+}^{2}$. We set $\epsilon_{c}=10$ and $\Delta \eta=0.2 / \mathcal{H}_{B+}$

oscillation, and it is blue shifted for small $k$ modes. We may analytically estimate it as follows.

For large $k$-modes, i.e. $k>\mathcal{H}_{B+}$,

$$
\begin{aligned}
\left|c_{3,1}-c_{3,2}\right|^{2} \approx & 1-\left(\frac{\mathcal{H}_{B+}}{k}-\frac{\alpha a_{B}^{2} \Delta \eta_{B}}{2 k}\right) \sin \left(\frac{2 k}{\mathcal{H}_{B+}}\right) \\
& -\left(\frac{\mathcal{H}_{B+}}{k}-\frac{\alpha a_{B}^{2} \Delta \eta_{B}}{2 k}\right) \\
& \times \sin \left(\frac{2 k}{\mathcal{H}_{B+}}+2 k \Delta \eta_{B}\right),
\end{aligned}
$$

which implies $P_{T}$ is flat and oscillating rapidly with the maximal amplitude at $k \simeq \mathcal{H}_{B+}$, just as showed in Figs. 2 and 3 . However, if the bounce phase lasts short enough, i.e. $\Delta \eta_{B} \sim$ 0 , (17) will be reduced to

$$
\left|c_{3,1}-c_{3,2}\right|^{2} \approx 1-\frac{2 \mathcal{H}_{B+}}{k} \sin \left(\frac{2 k}{\mathcal{H}_{B+}}\right) .
$$

For small $k$-modes, i.e. $k<\mathcal{H}_{B+}$,

$$
\begin{aligned}
&\left|c_{3,1}-c_{3,2}\right|^{2} \approx \frac{2^{\frac{2}{1-\epsilon_{c}}}}{\pi} \Gamma^{2}\left(\frac{1}{2}-\frac{1}{\epsilon_{c}-1}\right) \\
&\left(\epsilon_{c}-1\right)^{\frac{2}{\epsilon_{c}-1}} f\left(\Delta \eta_{B}\right) \\
&\left(\frac{k}{\mathcal{H}_{B+}}\right)^{\frac{2 \epsilon_{c}}{\epsilon_{c}-1}} \sim\left(\frac{k}{\mathcal{H}_{B+}}\right)^{\frac{2 \epsilon_{c}}{\epsilon_{c}-1}},
\end{aligned}
$$

which suggests that $P_{T} \sim\left(\frac{k}{\mathcal{H}_{B+}}\right)^{2}$ is strongly blue for $\epsilon_{C} \gg$ 1 , and $P_{T} \sim\left(\frac{k}{\mathcal{H}_{B+}}\right)^{3}$ for $\epsilon_{c} \simeq 3$. The result is consistent with that found in [19]. In (19),

$$
\begin{aligned}
f\left(\Delta \eta_{B}\right)= & {\left[\left(1-\frac{l^{2} \Delta \eta_{B}}{2 \mathcal{H}_{B+}}\right) \cosh \left(l \Delta \eta_{B}\right)+\frac{l}{2}\left(\frac{1}{\mathcal{H}_{B+}}-\Delta \eta_{B}\right.\right.} \\
& \left.\left.+\frac{l^{2}}{4 \mathcal{H}_{B+}} \Delta \eta_{B}^{2}\right) \sinh \left(l \Delta \eta_{B}\right)\right]^{2}
\end{aligned}
$$

However, if $\Delta \eta_{B} \sim 0, f\left(\Delta \eta_{B}\right) \sim 1$, and (19) will be reduced to

$$
\begin{aligned}
\left|c_{3,1}-c_{3,2}\right|^{2} \approx & \frac{2^{\frac{2}{1-\epsilon_{c}}}}{\pi} \Gamma^{2}\left(\frac{1}{2}-\frac{1}{\epsilon_{c}-1}\right) \\
& \times\left(\epsilon_{c}-1\right)^{\frac{2}{\epsilon_{c}-1}}\left(\frac{k}{\mathcal{H}_{B+}}\right)^{\frac{2 \epsilon_{c}}{\epsilon_{c}-1}} .
\end{aligned}
$$

\section{A realistic model of bounce inflation}

\subsection{Qiu-Wang model}

How to implement the bounce before inflation is still a significant issue. Recently, Qiu and Wang have proposed a realistic bounce inflation model without instabilities [15]. We briefly review it as follows.

The Lagrangian is

$$
\begin{aligned}
\mathcal{L}= & {\left[1-\frac{2 \gamma_{1}}{\left(1+2 \kappa_{1} \phi^{2}\right)^{2}}\right] X+\frac{\gamma_{2} X^{2}}{\left(1+2 \kappa_{2} \phi^{2}\right)^{2}} } \\
& -\frac{\gamma_{3} X}{\left(1+2 \kappa_{2} \phi^{2}\right)^{2}} \square \phi-V(\phi),
\end{aligned}
$$

where $X=-\partial_{\mu} \phi \partial^{\mu} \phi / 2$ and

$$
\begin{aligned}
V(\phi)= & -V_{0} e^{c \phi}\left[1-\tanh \left(\frac{\phi}{\lambda_{1}}\right)\right] \\
& +\Lambda^{4}\left(1-\frac{\phi^{2}}{v^{2}}\right)^{2}\left[1+\tanh \left(\frac{\phi}{\lambda_{2}}\right)\right],
\end{aligned}
$$

and $M_{P}=1$, and the values of parameters $\gamma_{1}, \gamma_{2}, \gamma_{3}, \kappa_{1}$, $\kappa_{2}, \lambda_{1}, \lambda_{2}, V_{0}, c, \Lambda$, and $v$ will determine the occurrence of bounce and inflation. 

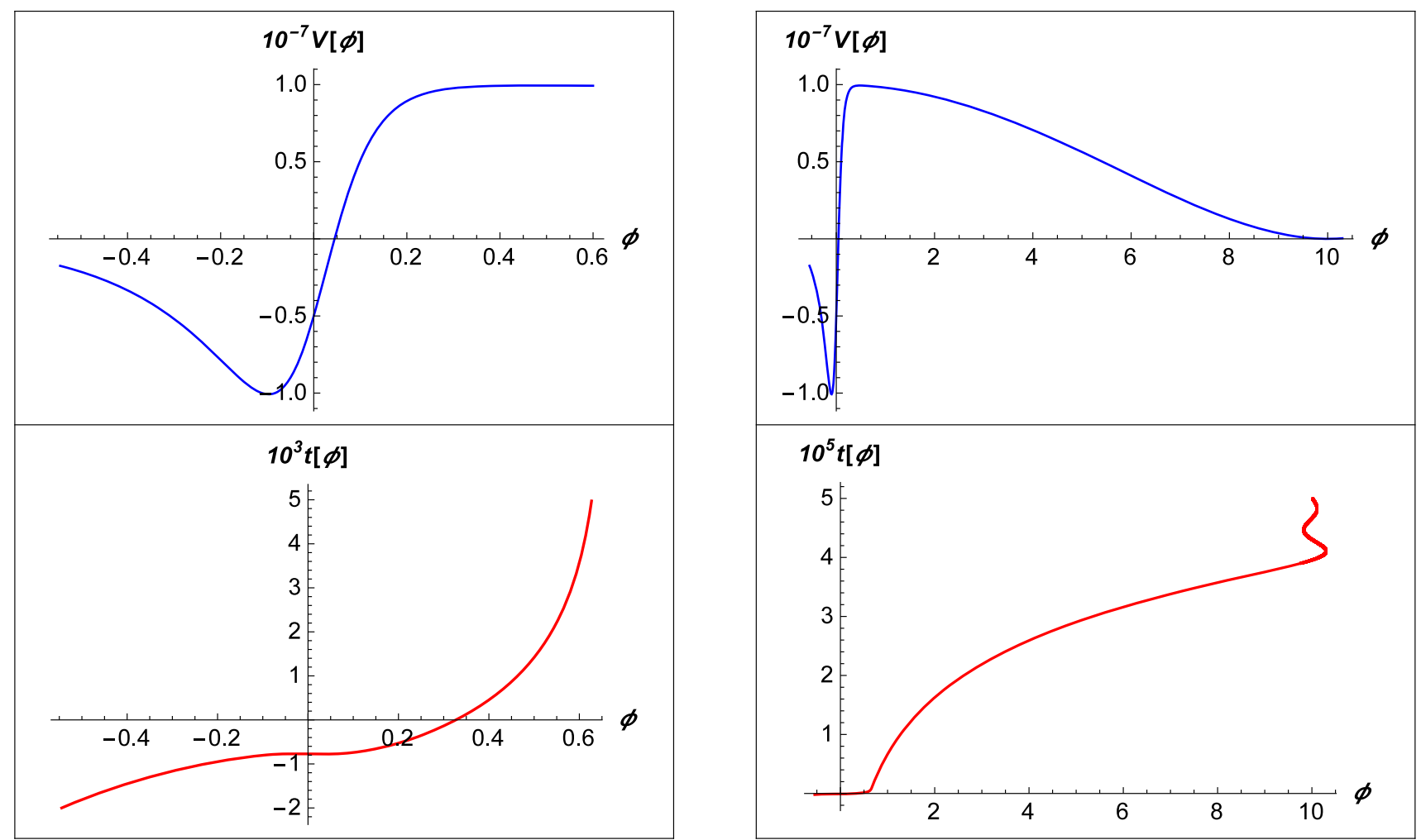

Fig. 4 Plots of potential $V(\phi)$ in (23), and the evolution of scalar field with respect to the physical time

The potential is plotted in the upper panels of Fig. 4. When $\phi \ll-\lambda_{1}, 1 / \sqrt{\kappa_{1}}, 1 / \sqrt{\kappa_{2}},(22)$ is

$\mathcal{L}_{c}=-\frac{\partial_{\mu} \phi \partial^{\mu} \phi}{2}+V_{0} e^{c \phi}$,

which will bring the ekpyrotic contraction with $\epsilon_{c}=c^{2} / 2>$ 3 . The bounce after the ekpyrotic contraction was also studied in [43]. When $\phi \gg-\lambda_{1}, 1 / \sqrt{\kappa_{1}}, 1 / \sqrt{\kappa_{2}}$, Eq. (22) reduces to that of slow-roll inflation,

$\mathcal{L}_{\text {inf }}=-\frac{\partial_{\mu} \phi \partial^{\mu} \phi}{2}-\Lambda^{4}\left(1-\frac{\phi^{2}}{v^{2}}\right)^{2}$

When $|\phi| \simeq 0$, Eq. (22) becomes ghost-like. However, there may be no instabilities, as has been confirmed in [15]; see also [44].

We plot the evolution of background in Figs. 5 and 6. The evolution of $\phi$ is plotted in Fig. 4. Initially, we require $\phi \ll$ $-\lambda_{1}, 1 / \sqrt{\kappa_{1}}, 1 / \sqrt{\kappa_{2}}$, and $\phi$ rolls down along its ekpyroticlike potential and the universe is contracting. When $t=t_{B-}$, the ekpyrotic contraction ends, and $\phi$ climbs up along its potential, which is essential so that inflation can occur subsequently, as showed originally in [6-8]. When $\phi$ arrives at $\phi \simeq 0$, the bounce will occur. Hereafter, $\phi$ continues to climb up to the potential hill, and then rolls slowly, and slow-roll

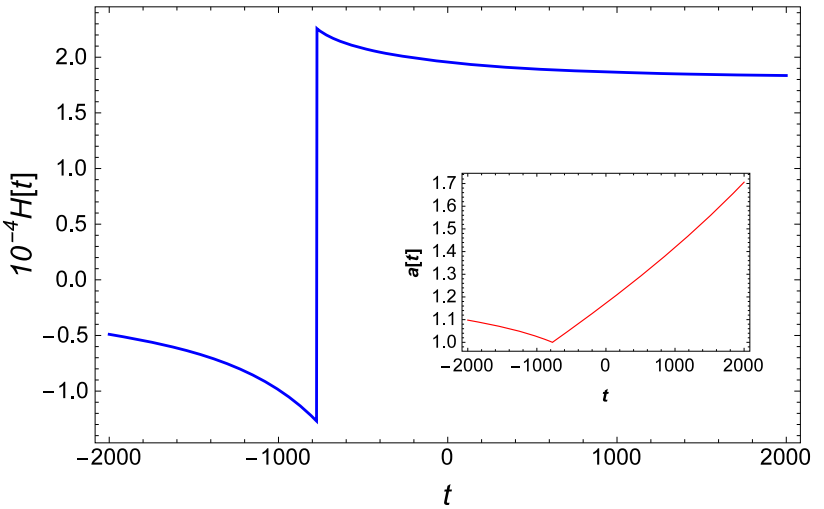

Fig. 5 The evolution of $a$ and $H=\dot{a} / a$ in the bounce inflation scenario, based on the model in Ref. [15], which will be showed in detail in Sect. 4

inflation starts. Reheating will occur around $\phi \simeq 10$, where $\phi$ oscillates and decays.

\subsection{The spectrum of primordial GWs: numerical result}

Here, with the background of Qiu-Wang model, which have been plotted in Figs. 5 and 6, we will numerically solve the perturbation equation (3) and plot the spectrum of the primordial GWs. 


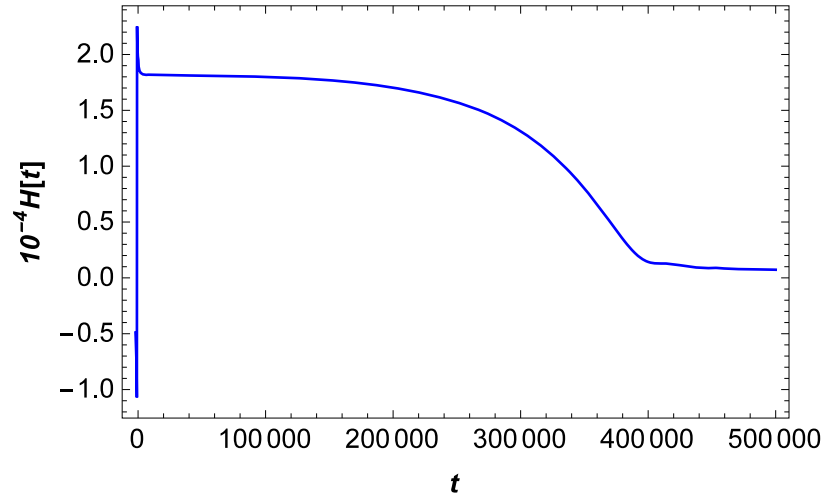

Fig. 6 The evolution of the Hubble parameter. We set the values of the parameters as $\gamma_{1}=0.6, \gamma_{2}=5, \gamma_{3}=10^{3}, \kappa_{1}=15, \kappa_{2}=10$, $\lambda_{1}=0.1, \lambda_{2}=0.1, V_{0}=0.7, c=\sqrt{20}, \Lambda=1.5 \times 10^{-2}$, and $v=10$

It is convenient for us to write the perturbation equation with respect to the physical time

$\frac{\mathrm{d}^{2} h_{k}}{\mathrm{~d} t^{2}}+3 H(t) \frac{\mathrm{d} h_{k}}{\mathrm{~d} t}+\frac{k^{2}}{a^{2}(t)} h_{k}=0$,

and considering $u_{k}=\frac{a h_{k}}{2}$, we have

$\frac{\mathrm{d}^{2} u_{k}}{\mathrm{~d} t^{2}}+H(t) \frac{\mathrm{d} u_{k}}{\mathrm{~d} t}+\left[\frac{k^{2}}{a^{2}(t)} u_{k}-H^{2}(t)-\frac{\ddot{a}}{a}\right] u_{k}=0$.

Initially we have

$u_{k}=\frac{1}{\sqrt{2 k}} e^{-i k \int \frac{\mathrm{d} t}{a}}, \quad \dot{u}_{k}=-i \sqrt{\frac{k}{2}} \frac{1}{a} e^{-i k \int \frac{\mathrm{d} t}{a}}$,

which suggest

$h_{k}=\frac{2}{\sqrt{2 k} a} e^{-i k \int \frac{\mathrm{d} t}{a}}, \quad \dot{h}_{k}=-2 \sqrt{\frac{2}{k}}\left(\frac{H(t)}{a}+\frac{i k}{a^{2}}\right) e^{-i k \int \frac{\mathrm{d} t}{a}}$.

We numerically plot $P_{T}$ in Figs. 7 and 11, which is completely consistent with our analytical result (15). Figure 11 tells us that the bounce phase must be short, otherwise the numerical curve will not overlap with the analytical one. However, Eq. (15) is actually universal, that is, independent of whether the bounce is short or not.

In addition, it should be mentioned that after the bounce, a period with $\dot{\phi}^{2}$ domination will appear; see Fig. 6 . This brief period has been often used to argue the suppression of a power spectrum, e.g., [16]. However, in bounce inflation scenario, such a period is actually only relevant with the oscillating of the spectrum, while the contraction before the bounce results in the suppression of a spectrum at large scale, as seen in Eqs. (17) and (19).

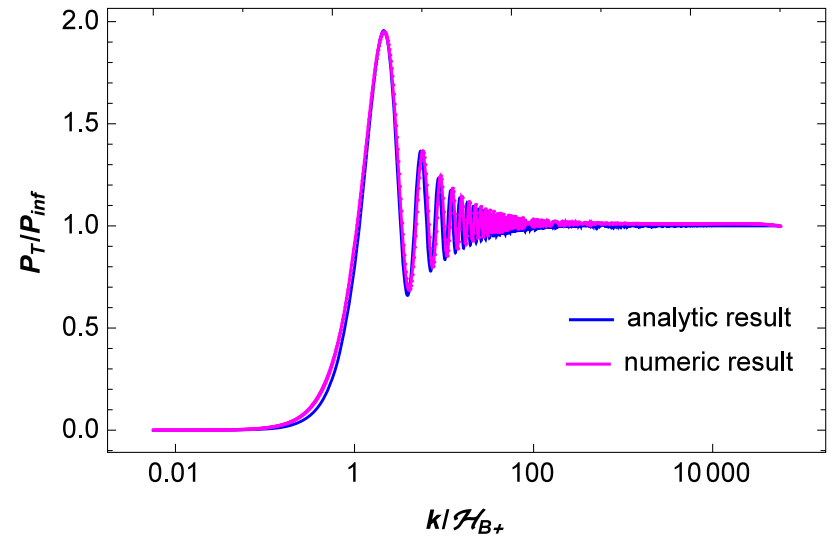

Fig. 7 The numeric GWs spectrum in the Qiu-Wang model is compared with our analytical result (15). We set $\epsilon_{C} \simeq 10, \alpha \sim 1.8 \times$ $10^{-3} \mathcal{H}_{B+}^{2}$, and $\triangle \eta_{B} \sim 0.2 / \mathcal{H}_{B+}$

\section{Discussion}

Bounce inflation is successful in solving the initial singularity problem of inflation and is also competitive for explaining the power deficit in CMB at large scale. This might provide us with an opportunity to comprehend the origin of inflation thoroughly. There are also other designs to explain the power deficit, such as [45-48], but these do not involve the initial singularity problem. The primordial GWs straightly encodes the evolution property of spacetime, thus it is interesting to have a detailed study of it.

We analytically calculated the bounce inflationary GWs. The resulting spectrum is written with the recursive Bogoliubov coefficients including the physics of the bounce phase. The spectrum acquires a large-scale cutoff due to the contraction, while the oscillation around the cutoff scale is quite drastic, which is determined by the details of the bounce. We also show that our analytic result is completely consistent with the numerical plotting for a realistic model of bounce inflation.

In original Refs. [6-8], the perturbation spectrum was calculated without considering the bounce phase, which is controversial, since conventionally it is thought that the bounce should affect the spectrum. However, we find that if the bounce phase lasts shortly enough, the effect of the bounce on the primordial GWs may be negligible, and the calculation without considering the bounce phase is robust.

We should point out that what we applied is the perturbation equation without modifying gravity, which may be implemented only when the null energy condition is broken. However, the physics of the bounce phase is unknown, it is obvious that the high-curvature corrections of gravity may also result in the occurrence of bounce, e.g., the GaussBonnet correction [49-52], and non-local gravity [53,54], which will inevitably modify the perturbation equation. We 
find that the corresponding corrections will aggravate the oscillating behavior around the cutoff scale $\mathcal{H}_{B+}$, however, the spectrum in the regime of $k \ll \mathcal{H}_{B-}$ and $k \gg \mathcal{H}_{B+}$ is hardly affected. We will come back to this issue in upcoming work.

In addition, as has been mentioned, the bounce inflation may also explain a large dipole power asymmetry in CMB at low $l$. However, the asymmetry might also appear in CMB $\mathrm{B}$-mode polarization [55,56]. Moreover, during the bounce, there might be a large parity violation [57]. It is interesting to have a reestimate for the relevant issues.

A series of experiments aiming at detecting GWs have been implemented or will be implemented. Our work suggests that searching primordial GWs at large scale might tell us if the bounce has ever happened before inflation.

Acknowledgements This work is supported by NSFC, Nos. 11222546, 11575188, 11690021, and the Strategic Priority Research Program of Chinese Academy of Sciences, No. XDB23010100.

Open Access This article is distributed under the terms of the Creative Commons Attribution 4.0 International License (http://creativecomm ons.org/licenses/by/4.0/), which permits unrestricted use, distribution, and reproduction in any medium, provided you give appropriate credit to the original author(s) and the source, provide a link to the Creative Commons license, and indicate if changes were made. Funded by SCOAP ${ }^{3}$.

\section{Appendix A: The effects of $u_{k}$ continuum and $h_{k}$ contin- uum on spectrum}

When we do calculations, it is convenience to write $h_{k}$ as $u_{k} / 2 a(\eta)$, since the evolution of $u_{k}$ satisfies a Bessel equation. However, the real GW mode is $h_{k}$, not $u_{k}$. Thus which of $u_{k}$ or $h_{k}$ is continuous on the matching surface might be a question that needs to be clarified. Our result (15) is based on the continuum of $h_{k}$, for which we will argue as follows.

We define the phase ' $i$ ' and ' $i+1$ ' as the conjoint phases, both are matched at $\eta_{0}$. The perturbations in different phases satisfy

$$
\left(\begin{array}{l}
h_{i} \\
h_{i}^{\prime}
\end{array}\right)=\left(\begin{array}{cc}
\frac{1}{a_{i}} & 0 \\
-\frac{a_{i}^{\prime}}{a_{i}^{2}} & \frac{1}{a_{i}}
\end{array}\right)\left(\begin{array}{c}
u_{i} \\
u_{i}^{\prime}
\end{array}\right),
$$

and

$$
\left(\begin{array}{l}
h_{i+1} \\
h_{i+1}^{\prime}
\end{array}\right)=\left(\begin{array}{cc}
\frac{1}{a_{i+1}} & 0 \\
-\frac{a_{i+1}^{\prime}}{a_{i+1}^{2}} & \frac{1}{a_{i+1}}
\end{array}\right)\left(\begin{array}{c}
u_{i+1} \\
u_{i+1}^{\prime}
\end{array}\right),
$$

where the prefactor 2 is neglected. The the continuum of $h_{k}$ and $\dot{h}_{k}$ at the matching surface $\eta_{0}$ suggest

$$
\left(\begin{array}{l}
h_{i} \\
h_{i}^{\prime}
\end{array}\right)=\left(\begin{array}{l}
h_{i+1} \\
h_{i+1}^{\prime}
\end{array}\right) \text {. }
$$

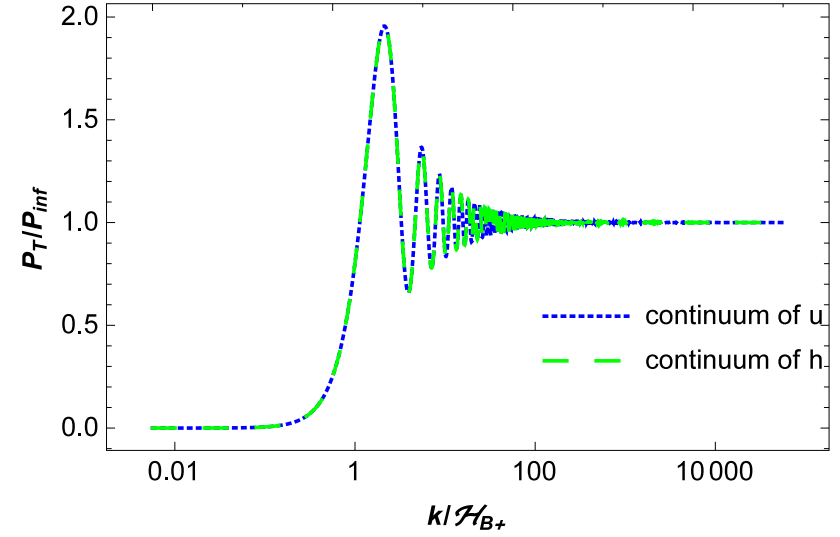

Fig. 8 The results are obtained by considering the continuity of $h_{k}$ (green curve) and $u_{k}$ (blue curve), with $\epsilon_{c} \simeq 10, \alpha \sim 1.8 \times$ $10^{-3} \mathcal{H}_{B+}^{2}$, and $\triangle \eta_{B} \sim 0.2 / \mathcal{H}_{B+}$

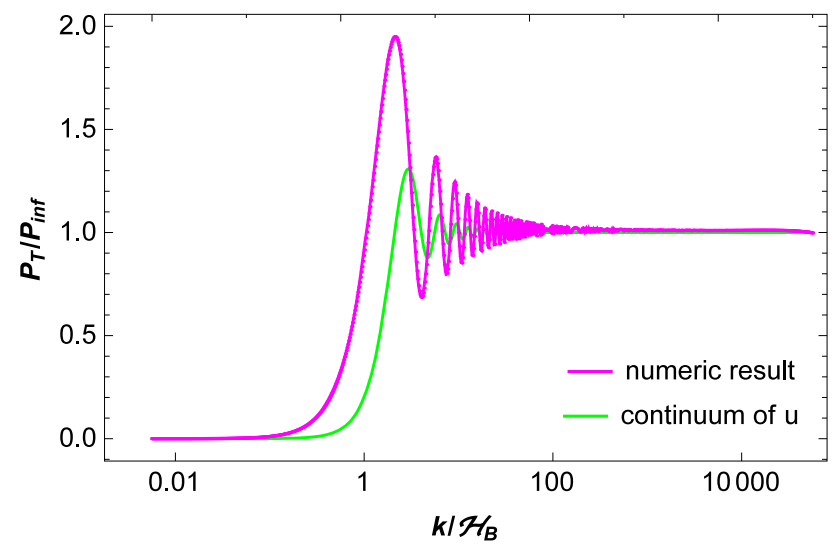

Fig. 9 The numeric result in Sect. 4 is compared with the analytical result, which is calculated by considering the continuity of $u_{k}$, with $\epsilon_{c} \simeq 10$

Thus we have

$\left(\begin{array}{c}u_{i+1} \\ u_{i+1}^{\prime}\end{array}\right)=\left(\begin{array}{cc}\frac{1}{a_{i+1}} & 0 \\ -\frac{a_{i+1}^{\prime}}{a_{i+1}^{2}} & \frac{1}{a_{i+1}}\end{array}\right)^{-1}\left(\begin{array}{cc}\frac{1}{a_{i}} & 0 \\ -\frac{a_{i}^{\prime}}{a_{i}^{2}} & \frac{1}{a_{i}}\end{array}\right)\left(\begin{array}{l}u_{i} \\ u_{i}^{\prime}\end{array}\right)$.

Finally,

$u_{i+1}=\frac{a_{i+1}}{a_{i}} u_{i}, \quad u_{i+1}^{\prime}=\left(\frac{a_{i+1}^{\prime}}{a_{i}}-\frac{a_{i+1} a_{i}^{\prime}}{a_{i}^{2}}+\frac{a_{i+1}}{a_{i}}\right) u_{i}^{\prime}$.

Generally, $a_{i+1}=a_{i}$ at the matching surface. Thus if $a_{i+1}^{\prime}=$ $a_{i}^{\prime}$, the continuum of $u$ is equal to that of $h$.

In Sect. 3.4, $a_{i+1}^{\prime}=a_{i}^{\prime}$ is ensured. We plot $P_{T}$ with the continuum of $u$ and $h$, respectively, in Fig. 8, which are completely identical. However, in Appendix B, the bounce phase is neglected, and we have $a^{\prime}\left(\eta_{B+}\right) \simeq-a^{\prime}\left(\eta_{B-}\right)$, which indicates that $a^{\prime}$ is not continuous. We analytically calculate $P_{T}$ with the continuum of $u$, and plot it in Fig. 9. It is found that 
the analytical result obtained cannot match with the numeric curve accurately.

\section{Appendix B: Is the effect of the bounce phase negligible?}

In the original idea of bounce inflation [6-8], the primordial GW spectrum was calculated by straightly jointing the perturbation solution in the contracting phase to that in the expanding phase. It is interesting to estimate if the effect of bounce phase is negligible.

We do not consider the bounce phase, which implies $\Delta \eta=0$ and $\mathcal{H}_{B+}=\mathcal{H}_{B-}=\mathcal{H}_{B}$. The spectrum of primordial GWs is still Eq. (15). However, $c_{3,1}$ and $c_{3,2}$ should be determined by straightly matching the solutions (7) and (14). The perturbation $\gamma_{i j}$ and its time derivative should be continuous through the match surface. Thus we have

$$
\left(\begin{array}{l}
c_{3,1} \\
c_{3,2}
\end{array}\right)=\mathcal{N}^{(3,1)} \times\left(\begin{array}{l}
c_{1,1} \\
c_{1,2}
\end{array}\right)
$$

with the matrix $\mathcal{N}^{(3,1)}$,

$$
\begin{aligned}
& \mathcal{N}_{11}^{(3,1)}=\frac{i \pi k \sqrt{x_{1} x_{2}}}{8}\left\{\left[H_{v_{1}+1}^{(1)}\left(k x_{1}\right)-H_{v_{1}-1}^{(1)}\left(k x_{1}\right)\right] H_{v_{2}}^{(2)}\left(k x_{2}\right)\right. \\
& +\left[H_{v_{2}-1}^{(2)}\left(k x_{2}\right)-H_{v_{2}+1}^{(2)}\left(k x_{1}\right)\right] H_{v_{1}}^{(1)}\left(k x_{1}\right) \\
& \left.+\frac{2}{k}\left(\frac{v_{2}}{x_{2}}-\frac{v_{1}}{x_{1}}\right) H_{v_{1}}^{(1)}\left(k x_{1}\right) H_{v_{2}}^{(2)}\left(k x_{2}\right)\right\} \text {, } \\
& \mathcal{N}_{12}^{(3,1)}=\frac{i \pi k \sqrt{x_{1} x_{2}}}{8}\left\{\left[H_{v_{1}+1}^{(2)}\left(k x_{1}\right)-H_{v_{1}-1}^{(2)}\left(k x_{1}\right)\right] H_{v_{2}}^{(2)}\left(k x_{2}\right)\right. \\
& +\left[H_{v_{2}-1}^{(2)}\left(k x_{2}\right)-H_{v_{2}+1}^{(2)}\left(k x_{1}\right)\right] H_{v_{1}}^{(2)}\left(k x_{1}\right) \\
& \left.+\frac{2}{k}\left(\frac{v_{2}}{x_{2}}-\frac{v_{1}}{x_{1}}\right) H_{v_{1}}^{(2)}\left(k x_{1}\right) H_{v_{2}}^{(2)}\left(k x_{2}\right)\right\} \text {, } \\
& \mathcal{N}_{21}^{(3,1)}=\frac{i \pi k \sqrt{x_{1} x_{2}}}{8}\left\{\left[H_{v_{1}-1}^{(1)}\left(k x_{1}\right)-H_{v_{1}+1}^{(1)}\left(k x_{1}\right)\right] H_{v_{2}}^{(1)}\left(k x_{2}\right)\right. \\
& +\left[-H_{v_{2}-1}^{(1)}\left(k x_{2}\right)+H_{v_{2}+1}^{(1)}\left(k x_{2}\right)\right] H_{v_{1}}^{(1)}\left(k x_{1}\right) \\
& \left.+\frac{2}{k}\left(\frac{\nu_{1}}{x_{1}}-\frac{\nu_{2}}{x_{2}}\right) H_{\nu_{1}}^{(1)}\left(k x_{1}\right) H_{\nu_{2}}^{(1)}\left(k x_{2}\right)\right\} \text {, } \\
& \mathcal{N}_{22}^{(3,1)}=\frac{i \pi k \sqrt{x_{1} x_{2}}}{8}\left\{\left[H_{v_{1}-1}^{(2)}\left(k x_{1}\right)-H_{v_{1}+1}^{(2)}\left(k x_{1}\right)\right] H_{v_{2}}^{(1)}\left(k x_{2}\right)\right. \\
& +\left[-H_{v_{2}-1}^{(1)}\left(k x_{2}\right)+H_{v_{2}+1}^{(1)}\left(k x_{2}\right)\right] H_{v_{1}}^{(2)}\left(k x_{1}\right) \\
& \left.+\frac{2}{k}\left(\frac{v_{1}}{x_{1}}-\frac{v_{2}}{x_{2}}\right) H_{v_{1}}^{(2)}\left(k x_{1}\right) H_{v_{2}}^{(1)}\left(k x_{2}\right)\right\} \text {. }
\end{aligned}
$$

For large $k$-modes, i.e. $k>\mathcal{H}_{B}$,

$$
\left|c_{3,1}-c_{3,2}\right|^{2} \approx 1-\frac{2 \mathcal{H}_{B}}{k} \sin \left(\frac{2 k}{\mathcal{H}_{B}}\right)
$$

which corresponds to (17). Meanwhile, for small $k$-modes, i.e. $k<\mathcal{H}_{B}$, the result is the same as (19).

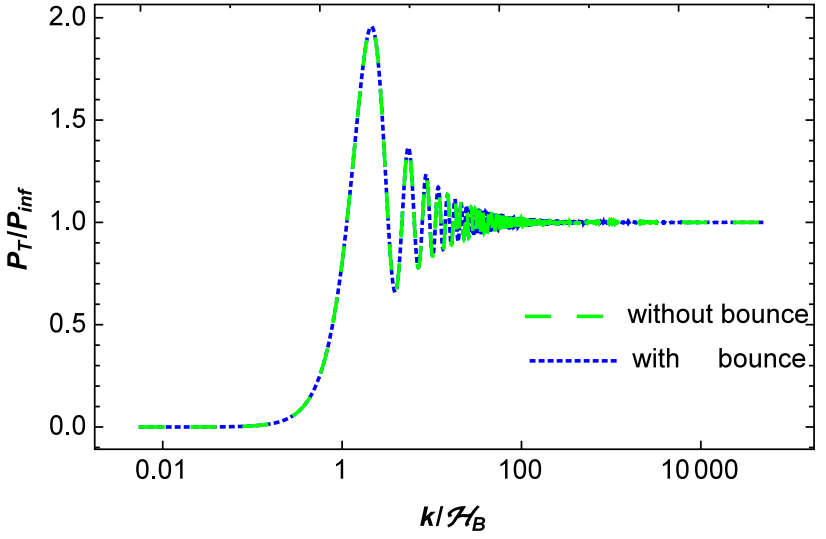

Fig. 10 The spectrum (15) is compared with that without considering the bounce phase. We set $\epsilon_{C} \simeq 10, \alpha \sim 1.8 \times 10^{-3} \mathcal{H}_{B+}^{2}$, and $\triangle \eta_{B} \sim$ $0.2 / \mathcal{H}_{B+}$

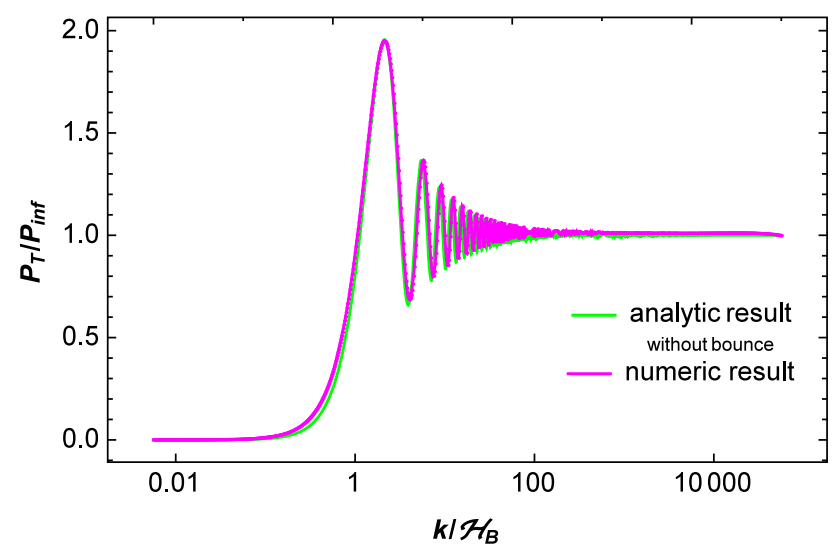

Fig. 11 The numeric GWs spectrum in Sect. 4 is compared with our analytical result (B1) without considering the bounce phase. $\epsilon_{c} \simeq 10$

In Fig. 10, we plot $P_{T}$ with (16) and (B1), respectively. Here, $\epsilon_{c} \simeq 10$. We see that if $\Delta \eta_{B} \sim 0.2 / \mathcal{H}_{B+}$ is set to parameterize the physics of the bounce, both curves completely overlap. This indicates that if the bounce lasts short enough, the effect of the bounce on the primordial GWs may be negligible. It is noticed in Sect. 3 that in a realistic model of bounce inflation, the period of the bounce is actually short enough, thus the result without considering the bounce phase is robust; see Fig. 11.

\section{References}

1. A.H. Guth, Phys. Rev. D 23, 347 (1981)

2. A.D. Linde, Phys. Lett. B 108, 389 (1982)

3. A. Albrecht, P.J. Steinhardt, Phys. Rev. Lett. 48, 1220 (1982)

4. A.A. Starobinsky, Phys. Lett. B 91, 99 (1980)

5. A. Borde, A. Vilenkin, Phys. Rev. Lett. 72, 3305 (1994). arXiv:gr-qc/9312022

6. Y.-S. Piao, B. Feng, X. Zhang, Phys. Rev. D 69, 103520 (2004). arXiv:hep-th/0310206 
7. Y.-S. Piao, Phys. Rev. D 71, 087301 (2005). arXiv:astro-ph/0502343

8. Y.-S. Piao, S. Tsujikawa, X. Zhang, Class. Quant. Grav. 21, 4455 (2004). arXiv:hep-th/0312139

9. T. Biswas, A. Mazumdar, Class. Quant. Grav. 31, 025019 (2014). arXiv: 1304.3648 [hep-th]

10. Z.G. Liu, Z.K. Guo, Y.S. Piao, Phys. Rev. D 88, 063539 (2013). arXiv: 1304.6527

11. F.T. Falciano, M. Lilley, P. Peter, Phys. Rev. D 77, 083513 (2008). arXiv:0802.1196 [gr-qc]

12. M. Lilley, L. Lorenz, S. Clesse, JCAP 1106, 004 (2011). arXiv: 1104.3494 [gr-qc]

13. J. Mielczarek, JCAP 0811, 011 (2008). arXiv:0807.0712 [gr-qc]

14. J.Q. Xia, Y.F. Cai, H. Li, X. Zhang, Phys. Rev. Lett. 112, 251301 (2014). arXiv:1403.7623 [astro-ph.CO]

15. T. Qiu, Y.T. Wang. arXiv:1501.03568 [astro-ph]

16. Y. Wan, T. Qiu, F.P. Huang, Y.F. Cai, H. Li, X. Zhang, JCAP 1512(12), 019 (2015). arXiv:1509.08772 [gr-qc]

17. P.A.R. Ade et al. [Planck Collaboration]. arXiv:1303.5062 [astroph.CO]

18. P.A.R. Ade et al. arXiv:1506.07135 [astro-ph.CO]

19. Y. Cai, Y.T. Wang, Y.S. Piao, Phys. Rev. D 92(2), 023518 (2015). arXiv:1501.01730 [astro-ph.CO]

20. D. Battefeld, P. Peter. arXiv:1406.2790 [astro-ph.CO]

21. J.L. Lehners, Class. Quant. Grav. 28, 204004 (2011). arXiv:1106.0172v1 [hep-th]

22. M.C. Guzzetti, N. Bartolo, M. Liguori, S. Matarrese. arXiv: 1605.01615 [astro-ph.CO]

23. T. Qiu, J. Evslin, Y.F. Cai, M. Li, X. Zhang, JCAP 1110, 036 (2011). arXiv:1108.0593 [hep-th]

24. T. Qiu, X. Gao, E.N. Saridakis, Phys. Rev. D 88, 4, 043525 (2013). arXiv:1303.2372 [astro-ph.CO]

25. D.A. Easson, I. Sawicki, A. Vikman, JCAP 1111, 021 (2011). arXiv:1109.1047 [hep-th]

26. M. Koehn, J.L. Lehners, B.A. Ovrut, Phys. Rev. D 90, 025005 (2014). arXiv:1310.7577 [hep-th]

27. L. Battarra, M. Koehn, J.L. Lehners, B.A. Ovrut, JCAP 1407, 007 (2014)

28. M. Koehn, J.L. Lehners, B. Ovrut, Phys. Rev. D 93, 10, 103501 (2016). arXiv:1512.03807 [hep-th]

29. J. Khoury, J.L. Lehners, B. Ovrut, Phys. Rev. D 83, 125031 (2011). arXiv:1012.3748 [hep-th]

30. M. Koehn, J.L. Lehners, B. Ovrut, Phys. Rev. D 87(6), 065022 (2013). arXiv:1212.2185 [hep-th]

31. Y.S. Piao, Phys. Rev. D 70, 101302 (2004). [arXiv:hep-th/0407258]

32. Y.S. Piao, Phys. Lett. B 677, 1 (2009). arXiv:0901.2644 [gr-qc]

33. Y.S. Piao, Phys. Lett. B 691, 225 (2010). arXiv:1001.0631 [hep-th]
34. J. Zhang, Z.G. Liu, Y.S. Piao, Phys. Rev. D 82, 123505 (2010). arXiv:1007.2498 [hep-th]

35. T. Biswas, S. Alexander, Phys. Rev. D 80, 043511 (2009). arXiv:0812.3182 [hep-th]

36. T. Biswas, A. Mazumdar, A. Shafieloo, Phys. Rev. D 82, 123517 (2010). arXiv:1003.3206 [hep-th]

37. W. Duhe, T. Biswas, Class. Quant. Grav. 31, 155010 (2014). arXiv:1306.6927 [astro-ph.CO]

38. T. Biswas, R. Mayes, C. Lattyak, Phys. Rev. D 93, 063505 (2016). arXiv: $1502.05875 \mathrm{v} 1$ [gr-qc]

39. S. Banerjee, E.N. Saridakis. arXiv:1604.06932v1 [gr-qc]

40. Y.-F. Cai, T. Qiu, Y.-S. Piao, M. Li, X. Zhang, JHEP 0710, 071 (2007). arXiv:0704.1090 [gr-qc]

41. Y.F. Cai et al., JCAP 0803, 013 (2008). arXiv:0711.2187 [hep-th]

42. J.K. Erickson, D.H. Wesley, P.J. Steinhardt, N. Turok, Phys. Rev. D 69, 063514 (2004). arXiv:hep-th/0312009

43. M. Osipov, V. Rubakov, JCAP 1311, 031 (2013). arXiv:1303.1221 [hep-th]

44. M. Libanov, S. Mironov, V. Rubakov. arXiv:1605.05992 [hep-th]

45. C.R. Contaldi, M. Peloso, L. Kofman, A.D. Linde, JCAP 0307, 002 (2003). arXiv:astro-ph/0303636

46. E. Dudas, N. Kitazawa, S.P. Patil, A. Sagnotti, JCAP 1205, 012 (2012). arXiv:1202.6630 [hep-th]

47. M. Bouhmadi-Lpez, P. Chen, Y.C. Huang, Y.H. Lin, Phys. Rev. D 87(10), 103513 (2013). arXiv:1212.2641 [astro-ph.CO]

48. M. Cicoli, S. Downes, B. Dutta, F. G. Pedro and A. Westphal. arXiv:1407.0148 [hep-th]

49. K. Bamba, A.N. Makarenko, A.N. Myagky, S.D. Odintsov, Phys. Lett. B 732, 349 (2014). arXiv:1403.3242 [hep-th]

50. K. Bamba, A.N. Makarenko, A.N. Myagky, S.D. Odintsov, JCAP 1504(04), 001 (2015). arXiv:1411.3852 [hep-th]

51. J. Haro, A.N. Makarenko, A.N. Myagky, S.D. Odintsov, V.K. Oikonomou, Phys. Rev. D 92(12), 124026 (2015). arXiv: 1506.08273 [gr-qc]

52. V.K. Oikonomou, Phys. Rev. D 92(12), 124027 (2015). arXiv: 1509.05827 [gr-qc]

53. T. Biswas, E. Gerwick, T. Koivisto, A. Mazumdar, Phys. Rev. Lett. 108, 031101 (2012). arXiv:1110.5249 [gr-qc]

54. T. Biswas, A.S. Koshelev, A. Mazumdar, S.Y. Vernov, JCAP 1208, 024 (2012). arXiv:1206.6374 [astro-ph.CO]

55. A.A. Abolhasani, S. Baghram, H. Firouzjahi, M.H. Namjoo, Phys. Rev. D 89(6), 063511 (2014). arXiv:1306.6932 [astro-ph.CO]

56. M.H. Namjoo, A.A. Abolhasani, H. Assadullahi, S. Baghram, H. Firouzjahi, D. Wands, JCAP 1505(05), 015 (2015). arXiv:1411.5312 [astro-ph.CO]

57. Y.T. Wang, Y.S. Piao, Phys. Lett. B 741, 55 (2015). arXiv:1409.7153 [gr-qc] 